\title{
Extração por orifícios naturais (NOSE) no tratamento cirúrgico minimamente invasivo de endometriose intestinal: relato de
} caso

\section{Natural orifice specimen extraction (NOSE) in minimally invasive surgical treatment of intestinal endometriosis: case report}

Letícia Matoso Freire ${ }^{1}$. Thaís Fontes de Magalhães ${ }^{1}$. Flora Cruz de Almeida ${ }^{1}$. Kathiane Lustosa Augusto ${ }^{2}$. Manuela Cavalcante Portela Marinho ${ }^{3}$. Carla Camila Rocha Bezerra ${ }^{4}$. Sthela Maria Murad Regadas ${ }^{5}$. Lusmar Veras Rodrigues ${ }^{6}$. Leonardo Robson Pereira Sobreira Bezerra ${ }^{7}$.

1 Acadêmica do curso de Medicina, Universidade Federal do Ceará (UFC), Fortaleza, Ceará, Brasil. 2 Mestre em Ciências MédicoCirúrgicas, Universidade Federal do Ceará (UFC), Fortaleza, Ceará, Brasil. 3 Médica da Maternidade Escola Assis Chateaubriand, Residência Médica em Ginecologia e Obstetrícia, Hospital Geral de Fortaleza, Fortaleza, Ceará, Brasil. 4 Residente de Coloproctologia, Hospital Universitário Walter Cantídio, Fortaleza, Ceará, Brasil. 5 Pós-doutorado na Cleveland Clinic, Flórida, Estados Unidos da América. Professora Associada do Departamento de Cirurgia, Universidade Federal do Ceará (UFC), Fortaleza, Ceará, Brasil. 6 Doutor em Medicina pela Universidade Federal do Ceará (UFC), professor do Departamento de Cirurgia da Universidade Federal do Ceará, Fortaleza, Ceará, Brasil. 7 Professor do Departamento Materno Infantil da Universidade Federal do Ceará (UFC), Doutor em Ginecologia, Universidade Federal de São Paulo.

\section{RESUMO}

A endometriose profunda é uma condição crônica que afeta mulheres jovens, muitas vezes resultando em dor e infertilidade. Quando há acometimento intestinal, o tratamento cirúrgico pode evoluir para a ressecção do segmento intestinal acometido, devido à extensão circunferencial e à profundidade da lesão. A cirurgia minimamente invasiva é uma alternativa adequada $\mathrm{e}$ apresenta menor tempo de hospitalização, menor índice de complicações e menos dor no pós-operatório. Buscando a manutenção do tamanho original das incisões cirúrgicas externas e a retirada do espécime cirúrgico, a técnica de extração de espécimes por orifícios naturais (NOSE - natural orifice specimen extraction) foi desenvolvida. Apesar de seus benefícios, tal técnica ainda é pouco utilizada para o tratamento da endometriose com acometimento intestinal. O objetivo deste relato de caso é demonstrar a viabilidade técnica da extração via vaginal de segmento de retossigmoide acometido com endometriose intestinal e discutir suas implicações e limitações.

Palavras-chave: Cirurgia endoscópica por orifício natural. Laparoscopia. Endometriose. Cirurgia videoassistida. Colpotomia. Mulheres.

\section{ABSTRACT}

Deep endometriosis is a chronic condition that affects young women, often resulting in pain and infertility. When there is intestinal involvement, surgical treatment can progress to resection of the affected bowel segment, due to circumferential extent and depth of the lesion. Minimally invasive surgery is a suitable alternative and results in shorter hospital stays, fewer complications and less pain during the postoperative period. The natural orifice specimen extraction (NOSE) technique was developed in order to remove the surgical specimen while maintaining the original size of external surgical incisions, despite its advantages, this technique is not widely used yet for the treatment of intestinal endometriosis. The purpose of this case report is to demonstrate the technical feasibility of the vaginal extraction of a rectosigmoid segment infiltrated by endometriosis and to discuss its implications and limitations.

Keywords: Natural orifice endoscopic surgery. Laparoscopy. Endometriosis. Video-assisted surgery. Colpotomy. Women.

Autor correspondente: Letícia Matoso Freire, Rua Alemanha, 822, Itapery, Fortaleza, Ceará. CEP: 60714-152. Telefone: +55 85985177464 .

E-mail: leticiamatoso@yahoo.com.br

Conflito de interesses: Não há qualquer conflito de interesses por parte de qualquer um dos autores.

Recebido em: 20 Out 2015; Revisado em: 20 Nov 2015; Aceito em: 11 Jan 2015. 


\section{INTRODUÇÃO}

Endometriose é uma condição crônica caracterizada pela implantação ectópica de tecido endometrial que afeta majoritariamente mulheres jovens, muitas vezes resultando em dor e infertilidade. $\mathrm{O}$ tratamento envolve uma combinação de terapias e requer acompanhamento a longo prazo devido ao seu caráter recidivante. ${ }^{1} \mathrm{O}$ tratamento cirúrgico de pacientes com endometriose tem sido um grande desafio, necessitando muitas vezes integrar técnicas com outras especialidades, como a coloproctologia e a urologia. ${ }^{2}$

$\mathrm{Na}$ endometriose profunda com acometimento intestinal, o tratamento cirúrgico pode evoluir para a ressecção do segmento acometido, dependendo de sua extensão circunferencial e de sua profundidade. A cirurgia minimamente invasiva como a laparoscopia traz benefícios bem estabelecidos em relação à dor no período pós-operatório, menor incisão cirúrgica, estética, tempo de hospitalização e incidência de complicações se comparada aos procedimentos abertos. ${ }^{3}$ Tradicionalmente, a ressecção intestinal laparoscópica no tratamento da endometriose envolve a expansão da incisão ou a realização de uma incisão de Pfannenstiel para a anastomose das alças e retirada do espécime cirúrgico, afetando os potenciais benefícios do procedimento laparoscópico., ${ }^{4,5}$

Em 1996, Rodwine descreveu uma alternativa para a extração de espécimes cirúrgicos em mulheres com endometriose profunda utilizando um orifício natural. A técnica de extração por orifícios naturais (NOSE - natural orifice specimen extraction), segundo o autor, diminuiria ainda mais o período de hospitalização, o tempo cirúrgico e a incidência de complicações em relação às cirurgias laparotômica e laparoscópica convencional. ${ }^{6}$ A NOSE já foi descrita no âmbito da cirurgia digestiva ${ }^{7}$ e para remoção de espécimes após uma histerectomia e esplenectomia combinada. ${ }^{8} \mathrm{O}$ uso da vagina e do ânus como portas para a cavidade peritoneal parece adequado para a ressecção e para a reconstrução do trânsito colorretal, tornando a cirurgia colorretal o campo ideal para a implementação da NOSE com bom risco-benefício. ${ }^{9,10}$

Apesar disso, a realização rotineira de NOSE no tratamento cirúrgico da endometriose profunda ainda não é disseminada. Este resumo demonstra a viabilidade técnica da extração via vaginal de segmento de retossigmoide acometido com endometriose intestinal e discute suas implicações e limitações.

\section{RELATO DE CASO}

Paciente F.R.C.D, 25 anos, apresentando dor pélvica intensa há 1 ano, associada à irregularidade do hábito intestinal, que se agravavam durante o período menstrual, acompanhada de hematoquezia e dispareunia profunda. Submetida a USG com mapeamento para endometriose com preparo intestinal, no qual se observou área nodular hipoecóica em parede anterior de alça sigmoide, medindo 2,6x1,2×2,8 cm comprometendo desde serosa até a camada muscular e $40 \%$ da circunferência da alça, e outra mal delimitada medindo $8,6 \times 1,3 \times 2,4 \mathrm{~cm}$ comprometendo desde serosa até camadas musculares e $50 \%$ da circunferência da alça. Procedeu-se à laparoscopia com o achado de extensa lesão em sigmoide, firmemente aderida às estruturas anexiais. Optou-se pela retossigmoidectomia parcial com anastomose término-terminal intracorpórea pela coloproctologia. A anastomose foi realizada com o uso de grampeador circular, em que grampos metálicos unem as porções terminais dos cotos do sigmoide e do reto, proporcionando hemostasia e compressão dos tecidos. Procedeu-se, então, a ressecção laparoscópica transvaginal combinada e a extração da peça cirúrgica.

\section{DISCUSSÃO}

A endometriose acomete de 5 a $15 \%$ das mulheres em idade reprodutiva, podendo acometer até $50 \%$ das mulheres

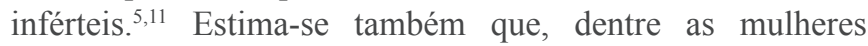
com endometriose, 3,8 a $37 \%$ irão apresentar envolvimento intestinal em algum momento. Caracteristicamente, a endometriose intestinal acomete o retossigmoide $(65,7 \%$ dos casos), causando diarreia, disquezia, cólica abdominal e dor ao defecar. ${ }^{12}$

Técnicas cirúrgicas minimamente invasivas vem gradualmente ganhando popularidade no tratamento da endometriose. Uma das limitações mais importantes da cirurgia endoscópica é a dificuldade de remover os espécimes cirúrgicos de diferentes tamanhos através de incisões muito pequenas. Esta etapa deve assegurar a recuperação completa da peça cirúrgica com a concomitante preservação da sua integridade.

A técnica clássica de ressecção colorretal por via laparoscópica envolve a realização de uma minilaparotomia ou alargamento de uma porta auxiliar por duas razões: a inserção de anvil para a anastomose mecânica e a extração do espécime cirúrgico. ${ }^{4}$ A utilização de um orifício natural para estas funções reduz significativamente a morbidade associada à cirurgia e, com o passar do tempo, diminui também o tempo intra-operatório. Pacientes submetidos à extração por NOSE também apresentam aumento significativo na satisfação com a imagem corporal após 35 meses de cirurgia. ${ }^{13}$ No caso apresentado, foi realizada a extração da peça por via transvaginal e a anastomose por grampeador circular inserido através do canal anal.

Utilizando-se a via anal para colocação do anvil, evitase a preparação do coto intestinal de forma extracorpórea, diminuindo assim a probabilidade de infecção perioperatória. ${ }^{4}$ Apesar de haver o risco teórico de contaminação peritoneal por translocação da flora bacteriana intestinal, Leroy e colaboradores mostraram que não houve aumento da morbidade por infecção com esta técnica. ${ }^{14}$

O uso da via transvaginal na NOSE é amplamente documentado. ${ }^{4,9}$ Alguns cirurgiões defendem que a realização de duas anastomoses adjacentes, na vagina e no reto, predispõe a fístulas reto-vaginais, e que portanto a extração vaginal de segmentos colônicos não seria recomendada. Entretanto, em uma revisão feita com 130 pacientes de 23 estudos, houve somente um caso de fístula vaginal atribuível à extração por 
NOSE. ${ }^{9}$ Uma outra revisão sistemática realizada por Wolthius em 2014 concluiu que a abertura proposital da vagina para entrada na cavidade peritoneal (colpotomia) não predispõe a dispareunia nem aumenta a morbidade pós-operatória, com o benefício adicional de causar menos dor no período pósoperatório. ${ }^{15}$ A mesma revisão sistemática mostra também que a extração transvaginal é possível mesmo nas ressecções colônicas à direita, situação que dificulta a extração transanal. Nos casos de endometriose em que há acometimento do fórnix da vagina e nas quais incisões colposcópica e retal já seriam realizadas para promover a remoção completa de lesões, a via transvaginal pode ser utilizada nos passos cirúrgicos subsequentes sem riscos adicionais. ${ }^{4}$

Além dos avanços nas técnicas cirúrgicas minimamente invasivas para o tratamento da endometriose, os avanços nos cuidados perioperatórios também contribuem na minimização de complicações e efeitos adversos. O desenvolvimento de programas de recuperação pós-operatória melhorada visam a

\section{REFERÊNCIAS}

1. Kennedy S, Bergqvist A, Chapron C, D’Hooghe T, Dunselman G, Greb R, et al. ESHRE guideline for the diagnosis and treatment of endometriosis. Hum Reprod. 2005;20(10):2698-704.

2. Lindsay SF, Luciano DE, Luciano AA. Emerging therapy for endometriosis. Expert Opin Emerg Drugs. 2005;20(3):449-61.

3. Aron M, Berger AK, Stein RJ, Kamoi K, Brandina R, Canes D, et al. Transvaginal nephrectomy with a multichannel laparoscopic port: a cadaver study. BJU Int. 2009;103(11):1537-41.

4. Akladios C, Faller E, Afors K, Puga M, Albornoz J, Redondo $\mathrm{C}$, et al. Totally laparoscopic intracorporeal anastomosis with natural orifice specimen extraction (NOSE) techniques, particularly suitable for bowel endometriosis. J Minim Invasive Gynecol. 2014;21(6):1095-102.

5. Spigolon DN, Amaral VF, Barra CM. Endometriosis: economic impact and its outlook. Femina. 2012;40(3):129-134.

6. Redwine DB, Koning M, Sharpe DR. Laparoscopically assisted transvaginal segmental resection of the rectosigmoid colon for endometriosis. Fertil Steril. 1996;65(1):193-7.

7. Nishimura A, Kawahara M, Honda K, Ootani T, Kakuta T, Kitami $\mathrm{C}$, et al. Totally laparoscopic anterior resection with transvaginal assistance and transvaginal specimen extraction: a technique for natural orifice surgery combined with reduced-port surgery. Surg Endosc. 2013;27(12):4734-40.

8. Ramalingam M, King J, Jaacks L. Transvaginal specimen extraction after combined laparoscopic splenectomy and hysterectomy: diminuição de morbimortalidade associada a procedimentos cirúrgicos e, junto com a laparoscopia e a NOSE, contribuem para a melhoria da recuperação e da qualidade de vida de pacientes com endometriose tratadas cirurgicamente. ${ }^{10}$

\section{CONCLUSÃO}

Em nosso meio, ainda há relutância por parte do cirurgião ginecológico em utilizar a "extração por orifícios naturais" (NOSE) para o tratamento da endometriose intestinal, talvez justificada pela falta de conhecimento acerca da baixa incidência de complicações. De fato, a "extração por orifícios naturais" (NOSE) representa uma técnica cirúrgica segura para o tratamento dessa entidade clínica, com a vantagem de possibilitar a manutenção do tamanho original das incisões cirúrgicas externas e a retirada do espécime cirúrgico através da vagina ou do ânus, contribuindo, assim, para a melhoria da recuperação e da qualidade de vida dos pacientes.

Introduction to NOSE (Natural Orifice Specimen Extraction) in a community hospital. Int J Surg Case Rep. 2013;4(12):1138-41.

9. Diana M, Perretta S, Wall J, Costantino FA, Leroy J, Demartines $\mathrm{N}$, et al. Transvaginal specimen extraction in colorectal surgery: current state of the art. Colorectal Dis. 2011;13(6):e104-11.

10. Kehlet H. Fast-track colorectal surgery. Lancet. 2008;371(9615):791-3.

11. Vigano P, Parazzini F, Somigliana E, Vercellini P. Endometriosis: epidemiology and aetiological factors. Best Pract Res Clin Obstet Gynaecol. 2004;18(2):177-200.

12. Meuleman C, Tomassetti C, D'Hoore A, Van Cleynenbreugel B, Penninckx F, Vergote I, et al. Surgical treatment of deeply infiltrating endometriosis with colorectal involvement. Hum Reprod Update. 2011;17(3):311-26.

13. Wolthuis AM, Meuleman C, Tomassetti C, D'Hooghe T, Fieuws $\mathrm{S}$, van Overstraeten $\mathrm{AB}$, et al. How do patients score cosmesis after laparoscopic natural orifice specimen extraction colectomy? Colorectal Dis. 2015;17(6):536-41.

14. Leroy J, Costantino F, Cahill RA, D’Agostino J, Morales A, Mutter D, et al. Laparoscopic resection with transanal specimen extraction for sigmoid diverticulitis. Br J Surg. 2011;98(9):1327-34.

15. Wolthuis AM, van Overstraeten AB, D'Hoore A. Laparoscopic natural orifice specimen extraction-colectomy: a systematic review. World J Gastroenterol. 2014;20(36):12981-92.

\section{Como citar:}

Freire LM, Magalhães TF, Almeida FC, Augusto KL, Marinho MC, Bezerra CC, et al. Extração por orifícios naturais (NOSE) no tratamento cirúrgico minimamente invasivo de endometriose intestinal: relato de caso. Rev Med UFC. 2016 jan-jun;56(1):49-51. 